\title{
Clinical Efficacy of a Dual Action, Topical Anti-edematous and Anti- inflammatory Device for the Treatment of External Hemorrhoids
}

Léa Shrivastava $^{1^{*}}$, Gisela da Silva Borges ${ }^{2}$ and Ravi Shrivastava ${ }^{1}$

${ }^{1}$ VITROBIO SAS and Research Institute, Issoire, France

${ }^{2}$ NATURVEDA SAS, Issoire, France

*Corresponding author: Léa Shrivastava, VITROBIO SAS and Research Institute, ZAC de Lavaur, 63500, Issoire, France, E-mail: 1s@vitrobio.com

Received date: January 12, 2018; Accepted date: February 09, 2018; Published date: February 13, 2018

Copyright: @ 2017 Léa Shrivastava, et al. This is an open-access article distributed under the terms of the Creative Commons Attribution License, which permits unrestricted use, distribution, and reproduction in any medium, provided the original author and source are credited.

\begin{abstract}
Objective: External hemorrhoids are enlarged, bulging blood vessels in and around the anus and lower rectum. They associate several pathologies such as engorged, edematous and inflamed sinusoids, with numerous proinflammatory cytokines on their surface, requiring a multi-target therapeutic approach. In the absence of any effective treatment, we assessed a newly conceived osmotically active, hypertonic, filmogen solution (Pileseptine-e) directed at attracting hypotonic liquid and helping suppress inflammation. The clinical efficacy and safety of Pileseptine-e on external hemorrhoids was evaluated in this study.
\end{abstract}

Methods: A 2-week treatment + 1-week follow-up, comparative, randomized, double blind, clinical trial with Pileseptine-e ( $n=37$, test product) versus saline spray $(n=17$, placebo) was performed in patients suffering from external hemorrhoids. Test and placebo products were applied as 3-4 sprays, 3-4 times per day, for 14 consecutive days. Parameters were evaluated employing a $0-4$ or $0-10$ scoring scale, before treatment (baseline, T0), $2 \mathrm{~h}$ after 1st treatment, and on Days 2, 3, 8, and 14, with follow-up check on Day 21.

Results: The test product induced an instant and strong outward exudation of liquid from inside the edematous hemorrhoids, thereby cleaning their surface, keeping it hydrated, and reducing pain and itching. A strong reduction in the size of hemorrhoids and rectal bleeding was also observed, which improved the quality of life of the patients considerably. The placebo product also provided noticeable symptomatic relief, but without effect on the size of hemorrhoids. No adverse effects were observed in any patient.

Conclusion: Reducing edema to allow hemorrhoidal volume to regress, and to normalize the structural physiology of the anal area, is the primary prerequisite to treat external hemorrhoids. Pileseptine-e is an antiedematous, cleaning, hydrating, safe and non-irritant filmogen solution that represents great advancement in the treatment of external hemorrhoids.

Keywords: External hemorrhoids; Filmogen; Osmotic; Antiedematous; Anti-inflammatory solution

\section{Introduction}

Hemorrhoids occur when the veins of the rectum or anus become dilated or enlarged. Depending on the location, they are considered "internal" or "external". External hemorrhoids are usually found beneath the skin that surrounds the anus as a lump around the anus (Figure 1).

External hemorrhoids are dilated varices of submucosal anal cushions and perianal venous plexus, distal to dentate line, affecting about $4-5 \%$ of the world population [1]. External hemorrhoids are most uncomfortable, because the overlying skin becomes irritated and erodes. If a blood clot forms inside an external hemorrhoid, the pain can be sudden and severe. The clot usually dissolves, leaving excess skin (a skin tag), which may itch or become irritated [2]. Wiping constantly to try to relieve the itching can worsen the problem. Due to their location at arteriovenous communication between terminal branches of rectal and hemorrhoidal arteries and veins of the hemorrhoidal plexus, poor efferent circulation leads to vascular dilation, vascular exudation, development of edema, and severe degradation of supporting tissues [3]. In the absence of any curative treatment, hemorrhoids tend to become chronic and inflamed, prone to thrombosis and ulceration. During the initial phase, antiinflammatory cytokines are secreted, particularly on the hemorrhoidal surface to suppress inflammation but if the process continues, then, pro-inflammatory cytokines are produced to maintain the inflammation [4]. After a few weeks, pro-inflammatory cytokines dominate an inflammatory cascade, and healing becomes extremely difficult $[5,6]$. Under such conditions, a logical treatment should be directed at eliminating edema, an essential step to minimize the size of the hemorrhoids and to normalize tissue physiology. The treatment should also simultaneously suppress inflammation to ease pain, irritation, itching, and bleeding. Healing of damaged tissue further requires keeping the tissue hydrated and infection-free. Unfortunately, it is practically impossible for a single drug to fulfill all these basic requirements at a time, which explains why there is no efficacious treatment for external hemorrhoids [4]. 


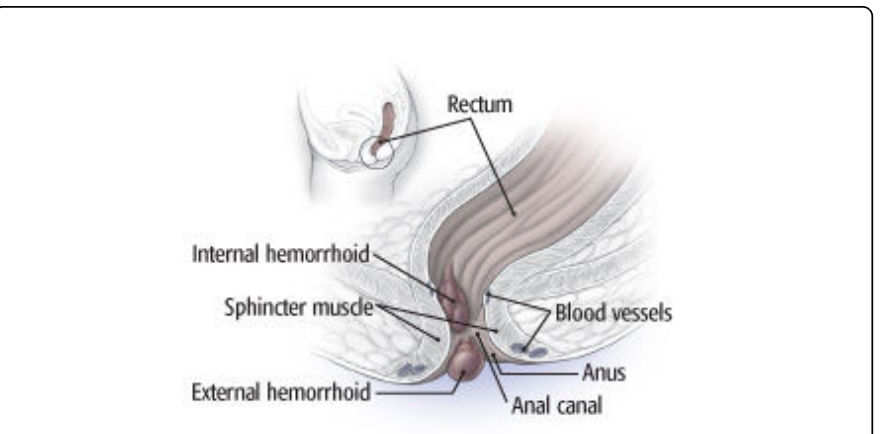

Figure 1: External hemorrhoids are dilated blood vessels on the perianal area, leading to the formation of edematous vascular lesion. They can be very uncomfortable and painful as the skin around them is irritated and often times requires medical attention.

Apart from surgical removal, in chronic cases, or ligatures, the most common treatments are topical anti-inflammatory drugs (steroids as cream, ointment, pad or suppository), analgesics (oral acetaminophen), topical anesthetics (pramoxine \& lidocaine), but these are all symptomatic treatments and may have side-effects $[7,8]$.

NATURVEDA SAS, a French pharmaceutical company, invented a glycerol-based, flexible liquid bandage (Pileseptine-e), relatively resistant to water dilution and capable of exerting a strong osmotic pressure over any semi-permeable live membrane filled with hypotonic liquid [9].

As the external hemorrhoidal wall is semi-permeable and is distended with excessive hypotonic liquid, it was purported that such a solution should attract hypotonic liquid from the inner parts of hemorrhoids to reduce their volume and to keep the outer hemorrhoidal surface hydrated, which should help lessen the pain, irritation and itching [10]. The aim of this study was therefore to evaluate the efficacy and safety of the new topical Pileseptine-e spray, containing an osmotically active hypertonic solution and polymers to render this solution filmogen, for the treatment of external hemorrhoids, compared to normal saline solution as placebo.

\section{Materials and Methods}

\section{Type of study}

This was a comparative, randomized, double blind, parallel group, observational clinical trial to evaluate the efficacy and safety of Pileseptine-e spray, containing pileseptine-e polymeric premix, versus isotonic saline solution as comparator/placebo, for the treatment of patients suffering from external hemorrhoids.

\section{Test product and placebo}

The test or treatment product (TP) was a viscous transparent liquid spray containing filmogen glycerol water, and pilecyanidine-e premix [11]. The pilecyanidine-e premix was an association of two glycerolbinding, natural plant polymers and a hydrophobic essential oil. The premix thus renders the glycerol base hydrophobic and filmogen upon application as a spray over the hemorrhoidal surface. The placebo spray or comparator product $(\mathrm{CP})$ contained $0.9 \% \mathrm{NaCl}$ in water and was physically identical to the TP with respect to color and consistency.
Both products were presented identically in $30 \mathrm{~mL}$ spray containers, with different identification codes for TP and CP.

\section{Study organizer}

The clinical part of this research was conducted at Nexus Clinical Research Center in India, affiliated to Nexus Clinical Research LLC, USA. The protocol and the study design were approved by the Institutional Ethical Committee of India - Rajiv Gandhi Institute of medical sciences (EC Registration $N^{\circ}$ ECR/492/Inst/AP/2013, dated 05/12/2013) and the trial was conducted following the ICH-GCP guidelines as per the declaration of Helsinki concerning ethical principles for medical research involving human subjects.

\section{Study design and rationales}

The primary objectives of the study were to evaluate the efficacy of the TP on external hemorrhoids, while the secondary objectives were to verify the safety and assess any eventual side effects of the TP. The doses were selected based on a previous pilot, dose range-finding, observational study on patients suffering from external hemorrhoids, where a nearly identical composition of polymeric glycerol was applied as a spray over the hemorrhoidal surface, 4 times per day for 14 days without any side effects. Saline solution was chosen as a placebo or comparator product as it is safe, non-hypertonic, non-irritant, and had physical characteristics nearly identical to the TP. Products were applied topically as a spray because this mode of application had been found very convenient by the patients during the pilot study.

\section{Inclusion and exclusion criteria}

At the time of recruitment at the study centers, patients were examined physically, and their medical, surgical, and allergic history was checked. Vital signs such as blood pressure, pulse rate, and respiratory rate were recorded. Patients not suffering from any serious pathology were then examined for enrollment in the study. The main inclusion criteria comprised: Patients with symptomatic uncomplicated external hemorrhoids without need of emergency operation according to the conclusion of the physical examination; ready to sign informed consent for participation in the study; males or females, aged 18-65 years; not under any treatment which may affect study outcome; and ready for manual rectal and proctological examinations as per study protocol. These inclusion criteria were chosen in order to obtain a homogenous population in the present study. It does not imply that the test product cannot be used by patients outside these criteria (e. g. the test product can be used by children above the age of 3 ). The key exclusion criteria were: hypersensitivity or allergy to any investigational product ingredients and pregnant or nursing women.

\section{Randomization}

After screening, patients satisfying all the inclusion criteria were enrolled and randomly allocated, in a 2:1 ratio, to either TP or CP group. Treatments were allocated to patient by carrying out randomization using SAS Version 9.1.3 following a randomization schedule. Block Randomization methodology was employed for generating the list (within the block the treatments were distributed in the ratio of 2:1). Each patient received a unique screening identification number, randomization code, and enrollment identification number. 


\section{Product presentation and administration}

TPs and CPs were supplied by NATURVEDA SAS Research Institute (ZAC de Lavaur 63500 Issoire, France) and were presented identically for blinding purpose $(30 \mathrm{~mL}$ sprays containing a viscous liquid) except for the product code and the batch number which were different.

Patients were asked to apply 3-4 sprays directly over the external hemorrhoidal surface, 3-4 times per day (morning, mid-day, evening, and before night rest) from Day 1 to Day 14 or till complete recovery (whichever was earlier). 1st treatment was administered just after the patient's inclusion in the study (Day 1). Although treatment was stopped on Day 14, patients were re-examined on Day 21 to evaluate the stability of the results or any rebound effects.

\section{Parameters studied}

while the secondary parameters included change in irbaseline, measured just before the start of the treatment): Visit 2 (Day 2), Visit 3 (Day 3), Visit 4 (Day 8), Visit 5 (Day 14 visit/end of therapy visit).

\section{Statistical analysis of results}

The analyses were conducted with Microsoft Excel and XLStat using the available data. Significant effects were those with a probability lower than $\alpha=0.05$. Statistically significant results were indicated in abbreviated manner: SS, and not statistically significant results ( $p>0.05)$ : NS. For each score, repeated measures analysis of variance (RMANOVA) determined differences in symptoms scores across study visits. Nemenyi post-hoc test provided pairwise comparisons in a group between baseline and the end of the study. Results were compared with baseline values (T0, scores obtained just before 1st treatment on Day 1) in the same group and with the $\mathrm{CP}$, at each time point.

\section{Results}

\section{Study population}

Out of 63 patients enrolled in the study ( $48 \mathrm{M}$ and 15F, mean age 38 years), 57 were randomized: 19 patients in the $\mathrm{CP}$ group and 38 in the TP group were dosed, and after drop-outs, 17 patients in the CP group and 37 in the TP group completed the study (Figure 2). No patient was excluded or dropped-out during the dosing period due to any unwanted side effects.

Overall, the baseline mean symptom scores were fairly identical in both groups, with exception for the lesion hydration and defecate discomfort parameters, with mean severity slightly higher in the CP group at study outset. This difference did not biased the effect of the treatment with TP

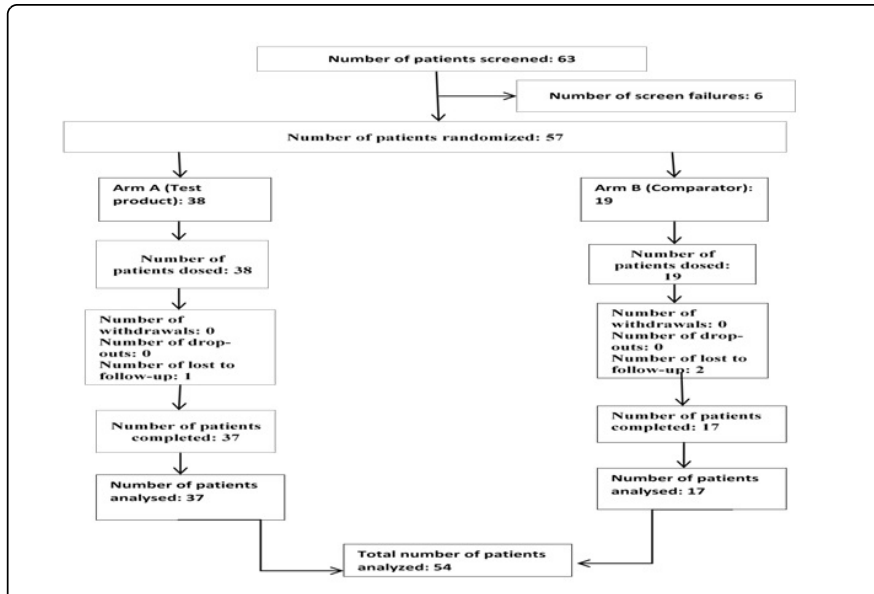

Figure 2: Flow chart of the study population.

\section{Effect on the size/volume of hemorrhoids}

The results are shown in Table 1 and Figure 3. During the 14-day treatment period, the size of the hemorrhoids in $\mathrm{CP}$ group slightly decreased (less than 10\%; Figure 3). When analyzing in the detail the results at the end of the study (day 14; Table 1), among the $17 \mathrm{CP}$ group patients, the size of the hemorrhoids was decreased slightly (less than $10 \%$ at all time-points compared to $\mathrm{T} 0$ values) in $11 / 17$ patients, strongly in 1 patient ( $\left.\mathrm{N}^{\circ} 35\right)$, while an increase was observed in $5 / 17$ patients. Among these 5 patients, the increase was relatively strong in 1 patient $\left(\mathrm{N}^{\circ} 53\right)$. During These results indicate that the placebo product does not affect the volume of the hemorrhoids even after 14 days of regular application, confirming that saline solution, being isotonic, is not capable of extracting liquid from inside the hemorrhoids, although it helps humidify the lesion and prevent hemorrhoidal enlargement in most of the patients.

\begin{tabular}{|l|l|l|l|l|l|}
\hline & $\begin{array}{l}\text { Increase } \\
\mathrm{d}\end{array}$ & $\begin{array}{l}\text { Remained } \\
\text { stable }\end{array}$ & $\begin{array}{l}\text { Decreas } \\
\text { es }\end{array}$ & $\begin{array}{l}\text { Strongly } \\
\text { decreased }\end{array}$ & $\begin{array}{l}\text { Nearly } \\
\text { disappeared }\end{array}$ \\
\hline $\begin{array}{l}\mathrm{CP} \\
(\mathrm{n}=17)\end{array}$ & 5 & 0 & 11 & 1 & 0 \\
\hline$\%$ & 0.2941 & 0 & 0.6471 & 0.0588 & 0 \\
\hline $\begin{array}{l}\text { TP } \\
(\mathrm{n}=37)\end{array}$ & 4 & 2 & 10 & 17 & 4 \\
\hline$\%$ & 0.1081 & 0.0541 & 0.2702 & 0.4595 & 0.1081 \\
\hline
\end{tabular}

Table 1: Effect on the size/volume of external hemorrhoids at the end of the study (day 14). The results are expressed as the number and \% of patients with increase or decrease in the size in the placebo $(n=17)$ and TP $(n=37)$ groups compared to the T0 baseline values.

In the TP-Pileseptine-e treated group, during the 14-day treatment period, the mean reduction was of $19.96 \%$ at $2 \mathrm{~h}, 6.45 \%$ on Day 2, $29.44 \%$ on Day 3, $35.89 \%$ on Day 8 and $48.29 \%$ on Day 14 , compared to baseline mean value (Figure 3). Despite treatment being stopped on Day 14 , hemorrhoid size was $52.02 \%$ lesser on Day 21 compared to pre-treatment, as the results obtained during the treatment period remained stable and even further improved during the 1-week followup period. Compared to the $\mathrm{CP}$ group, the reduction in the TP group 
was higher by $20 \%, 5.6 \%, 25 \%, 33 \%, 45 \%$ and $48 \%$ on Days $1,2,3,8$, 14 , and 21 , respectively (SS: $\mathrm{p}<0.01$ vs $\mathrm{CP}$ on day 3 and $\mathrm{p}<0.001$ vs $\mathrm{CP}$ from day 8 onwards). When analyzing in the detail the results at the end of the study (day 14; Table 1), the size of the hemorrhoids slightly increased in 4/37 and remained nearly unchanged in $2 / 37$ patients at the end of the study compared to the starting values. A relatively good reduction in size/volume was noticed in $10 / 37$ patients while the reduction was very strong in $17 / 37$ patients ( $>45 \%)$. In the remaining 4 patients, hemorrhoids had nearly disappeared by the end of the treatment. These results clearly indicate a strong anti-edematous effect of the TP, due to the removal of hypotonic liquid from inside the hemorrhoid (Figure 3). As the anti-edematous effect was much stronger during the 1st week of treatment (up to Day 8) and slowed down slightly thereafter, it is suggested to continue the treatment for at least 1 month in order to obtain nearly total recovery. It is also interesting to note that the volume of the hemorrhoids was reduced very strongly $(-20 \%)$ just $2 \mathrm{~h}$ after 1 st $\mathrm{TP}$ application but the hemorrhoids resumed nearly their initial volume on Day $2(-5.6 \%$ compared to $\mathrm{T} 0$ and $-0.5 \%$ compared to $\mathrm{CP}$ at the same time point) and again started decreasing strongly and progressively thereafter, up to Day 14 (Figure 3). This effect is probably related to the fact that 1 st volume/size was measured exactly $2 \mathrm{~h}( \pm 15 \mathrm{~min})$ after 1 st application of TP, during the period when TP film is highly active over the hemorrhoidal surface with strong anti-edematous effects leading to a strong reduction in size during those first $2-3 \mathrm{~h}$. As interstitial liquid continues pouring during the subsequent dilution of the film, before the next application, it probable leads to an increase in size of hemorrhoid, temporarily reversing the initial effect. The size starts decreasing permanently when all edematous liquid is extracted from the hemorrhoid.

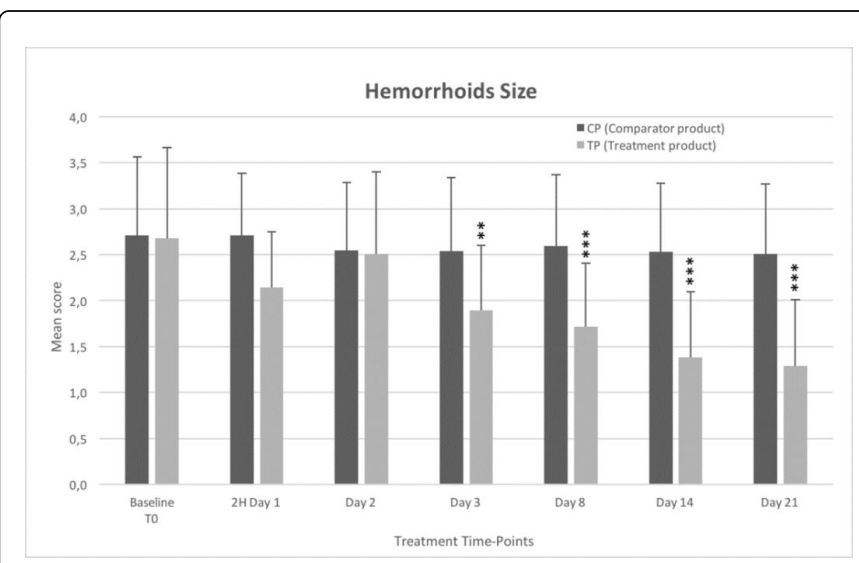

Figure 3: Effect of Pileseptine-e (TP) on the hemorrhoidal size/ volume scores quantified on a 0 to 4 rating scale at the start of the treatment (baseline T0), $2 \mathrm{~h}$ after 1st treatment, and on days $2,3,8$, and 14 as well as at the end of the follow-up period (day 21) in the TP (gray bars) and in the CP (black bars) groups. Values presented as Mean \pm S.D.. Statistical significance (SS) is indicated by ${ }^{* *} \mathrm{p}<0.01$ and ${ }^{* * *} \mathrm{p}<0.001$ for TP compared to CP at the same time point.

\section{Effect on the intensity of pain}

The initial scores for pain intensity was 7.29/10 in the CP group and $7.22 / 10$ in the TP group, evolving to $5.41 / 10$ in the $\mathrm{CP}$ group and $1.35 / 10$ in the TP group on Day 21. In the CP group, pain intensity was not affected $2 \mathrm{~h}$ after first treatment, but was reduced by $7.26 \%, 14.52 \%$,
$18.55 \%, 24.19 \%$, and $25.81 \%$ on Days $2,3,8,14$, and 21 , respectively (statistically significant on Days 8,14 , and 21 ; SS: $\mathrm{p}<0.05$ compared to baseline T0). These results show that regular application of CP helps diminish hemorrhoid pain to some extent but this reduction is slight, although it is progressive and does remain stable during the follow-up period after the treatment is stopped (Figure 4).

In the TP group, the mean pain score was diminished by $14.23 \%$ just $2 \mathrm{~h}$ after $1 \mathrm{st}$ treatment as compared to baseline value (13.76\% compared to $\mathrm{CP}$ ). Although statistically not significant, this early analgesic effect is highly encouraging and proves the osmotic properties of the TP. From Day 2 onward, the reduction in pain intensity was marked, progressive, and highly significant up to the end of the observation period (SS: $\mathrm{p}<0.05$ compared to baseline T0). Compared to the CP group, the reduction was higher by $23.29 \%, 32.81 \%, 45.87 \%$, and $62.36 \%$, respectively on Days 2, 3, 8, and 14. Surprisingly, this reduction continued up to Day 21 , when the mean value shows a $75.03 \%$ stronger decrease compared to the CP (SS; $\mathrm{p}<0.001$, TP compared to $\mathrm{CP}$ ). These results correspond to, and are probably related to, the reduction in size/volume of the lesions observed in the TP group.

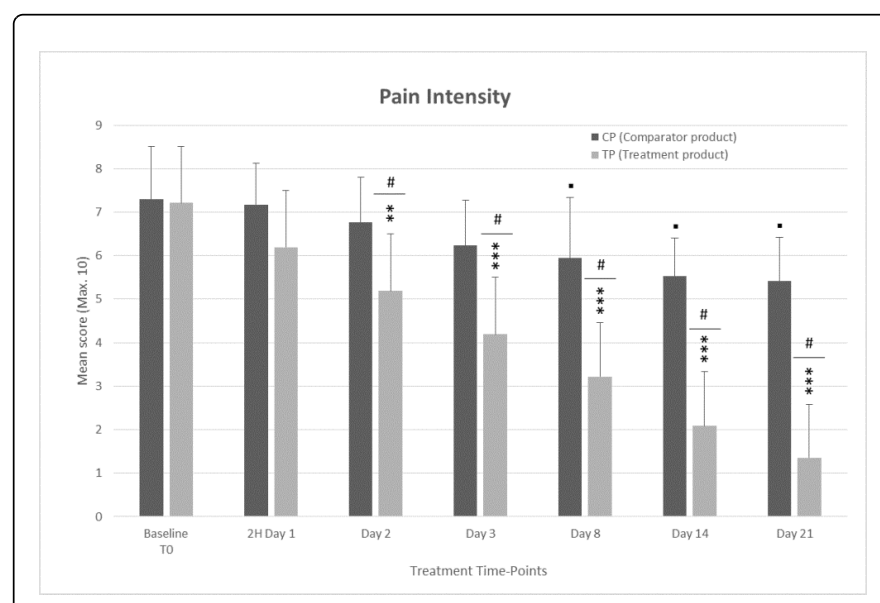

Figure 4: Effect of Pileseptine-e (TP) on the pain intensity scores quantified on a 0 (no pain) to 10 (highly painful) rating scale at the start of the treatment (baseline T0), $2 \mathrm{~h}$ after 1st treatment, and on days 2, 3, 8, and 14 as well as at the end of the follow-up period (day 21 ) in the CP (black bars) and in the TP (gray bars) groups. Values presented as Mean \pm S.D.. Statistical significance (SS) is indicated by ${ }^{* *} \mathrm{p}<0.01$ and ${ }^{* * *} \mathrm{p}<0.001$ for TP compared to CP at the same time point, $\mathrm{p}<0.05$ compared to baseline values for $\mathrm{CP}$, \# $\mathrm{p}<0.05$ compared to baseline values for TP.

\section{Effect on hemorrhoidal itching and irritation}

Mean itching intensity was reduced in both groups, but only $29 \%$ of the patients experienced regular decrease in the $\mathrm{CP}$ group with no effect in the first $2 \mathrm{~h}$, and $8.77 \%, 15.79 \%, 21.93 \%$, and $27.19 \%$ decrease, on Days 2, 3, 8, and 14, respectively (SS: $\mathrm{p}<0.05$ on Days 3, 8, and 14 compared to baseline T0). Reduction was only of $20.18 \%$ at the followup visit compared to baseline (SS: $\mathrm{p}<0.05$ ), indicating that the minor reduction in irritation observed with the $\mathrm{CP}$ during the treatment period tended to regress during the untreated follow-up period (Figure $5)$. 
In the TP group, an anti-irritant effect was seen just $2 \mathrm{~h}$ after $1 \mathrm{st}$ treatment $(-14.34 \%$ compared to baseline T0). This effect was significant when compared to the CP group (SS: $\mathrm{p}<0.05$ ). From Day 2 onward, the soothing but progressive and significant effect was observed with stronger reduction by $19.15 \%$ on Day $2,30.12 \%$ on Day $3,43.73 \%$ on Day 8 , and $57.93 \%$ on Day 14 , compared to CP scores (SS: $\mathrm{p}<0.01$ for Day 2 and $\mathrm{p}<0.001$ Day 3 and onwards). The anti-irritant effect further progressed on Day $21(-74.25 \%$; SS: $p<0.001)$, this longlasting effect indicating a curative effect of the TP. Only $5 \%$ patients in the TP group, compared to $95 \%$ in the CP group, complained about higher irritation on Day 21 than on Day 14. The itching sensation had totally disappeared for $24 \%$ of patients in the TP group while it remained at relatively high levels in the $\mathrm{CP}$ group, indicating that the TP strongly alleviate hemorrhoidal irritation (Figure 5).

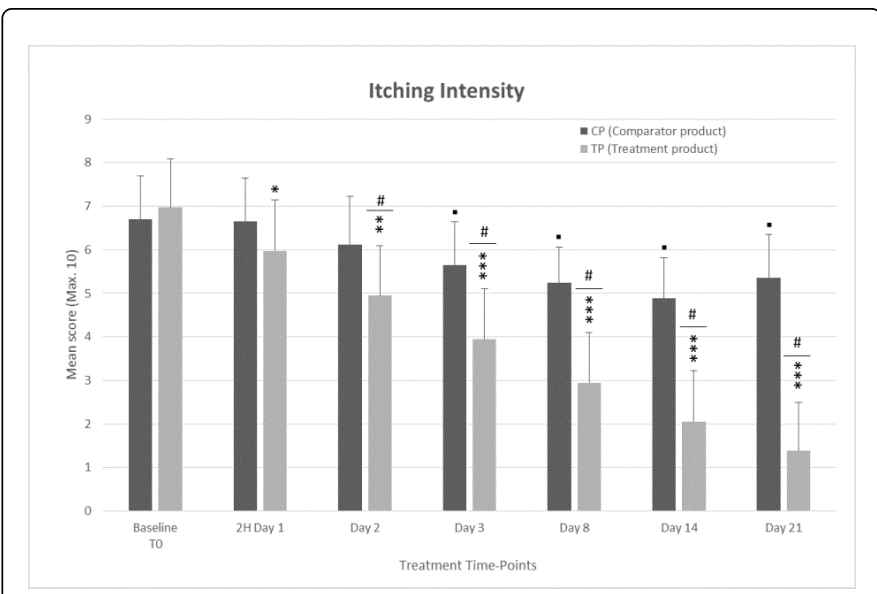

Figure 5: Effect of Pileseptine-e (TP) on the sensation of itching score quantified on a 0 (no symptom) to 10 (severe itching) rating scale at the start of the treatment (baseline T0), $2 \mathrm{~h}$ after 1 st treatment, and on days $2,3,8$, and 14 as well as at the end of the follow-up period (day 21) in the CP (black bars) and in the TP (gray bars) groups. Values presented as Mean \pm S.D.. Statistical significance (SS) is indicated by ${ }^{*} \mathrm{p}<0.05,{ }^{* *} \mathrm{p}<0.01$ and ${ }^{* * *} \mathrm{p}<0.001$ for TP compared to CP at the same time point, $\mathrm{p}<0.05$ compared to baseline values for $\mathrm{CP}, \# \mathrm{p}<0.05$ compared to baseline values for TP. NB: Identical results were obtained for the measurements of irritation after defecation (data not shown).

Similar results were obtained regarding the mean score for irritation after defecation. Indeed the TP group showed greater improvements in this parameter from Day 2 and onwards $(\mathrm{p}<0.001$ compared to CP; Data not shown).

\section{Effect on rectal bleeding}

The initial scores for rectal bleeding was $6.71 / 10$ in the CP group and 6.35/10 in the TP group, evolving to 5.47/10 in the CP group and $1.22 / 10$ in the TP group on Day 21. In the CP group, rectal bleeding was reduced by $5.26 \%, 12.28 \%, 16.67 \%, 22.81 \%$, and $18.42 \%$ on Days 2 , $3,8,14$, and 21 , respectively (SS: $\mathrm{p}<0.05$ Days 8,14 , and 21 compared to baseline T0). These results show that regular application of CP helps diminish rectal bleeding to some extent but this reduction is slight, although it is progressive and tended to regress during the untreated follow-up period after the treatment is stopped (Figure 6).
In the TP group, the mean rectal bleeding score was diminished by $23.83 \%$ just $2 \mathrm{~h}$ after 1 st treatment as compared to baseline value although statistically not significant and $23.85 \%$ compared to CP (SS: $\mathrm{p}<0.001$ ). From Day 2 onward, the reduction in rectal bleeding was marked, progressive, and highly significant up to the end of the observation period (SS: $\mathrm{p}<0.05$ compared to baseline T0). Compared to the CP group, the reduction was higher by $23.85 \%, 43.95 \%, 58.89 \%$, and $75.46 \%$, respectively on Days $2,3,8$, and 14 . Surprisingly, this reduction continued up to Day 21 , when the mean value shows a $77.77 \%$ stronger decrease compared to the CP (SS: $\mathrm{p}<0.001 \mathrm{TP}$ compared to $\mathrm{CP}$ ).

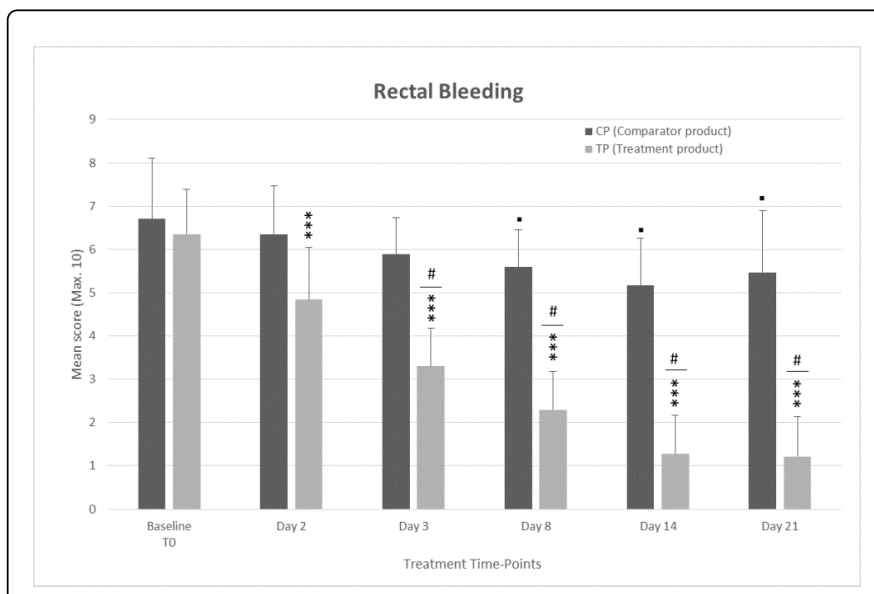

Figure 6: Effect of Pileseptine-e (TP) on the rectal bleeding scores quantified on a 0 (no bleeding) to 10 (severe bleeding) rating scale at the start of the treatment (baseline T0), on days 2, 3, 8, and 14 as well as at the end of the follow-up period (day 21) in the TP (gray bars) and in the CP (black bars) groups. Values presented as Mean \pm S.D.. Statistical significance (SS) is indicated by ${ }^{* * *} \mathrm{p}<0.001$ for TP compared to $\mathrm{CP}$ at the same time point, $\mathrm{p}<0.05$ compared to baseline values for $\mathrm{CP}, \# \mathrm{p}<0.05$ compared to baseline values for TP.

\section{Effect on lesion hydration and defecate discomfort}

Mean lesion (de)hydration score was reduced in the CP group with no effect in the first 2 days, and $10.08 \%, 16.81 \%$, and $20.17 \%$ decrease, on Days 3, 8, and 14, respectively (SS:p $<0.05$ on Days 8, and 14 compared to baseline T0). Reduction was only of $19.33 \%$ at the followup visit compared to baseline ( $S S: p<0.05)$, indicating that the minor improvement in lesion hydration observed with the CP during the treatment period tended to regress during the untreated follow-up period (Figure 7A).

In the TP group, an improvement in lesion hydration was seen from Day 2 onward, with stronger scores reduction by $36.23 \%$ on Day 2, $48.04 \%$ on Day $3,71.69 \%$ on Day 8 , and $85.01 \%$ on Day 14 , compared to CP scores (SS:p $<0.001$ for Day 2 and onwards). The progression in the lesion hydration improvement was not observed in the follow-up period but was still greater and highly significant than the observed in the CP group $(-19.33 \%$ in the $\mathrm{CP}$ and $-83.94 \%$ in the TP group, SS:p<0.001) (Figure 7A).

Similar results were obtained regarding the mean score for defecation discomfort. Indeed the $\mathrm{TP}$ group showed greater improvements in this parameter from Day 2 and onwards (SS:p $<0.001$ compared to CP from Day 2 onwards; Figure 7B). 


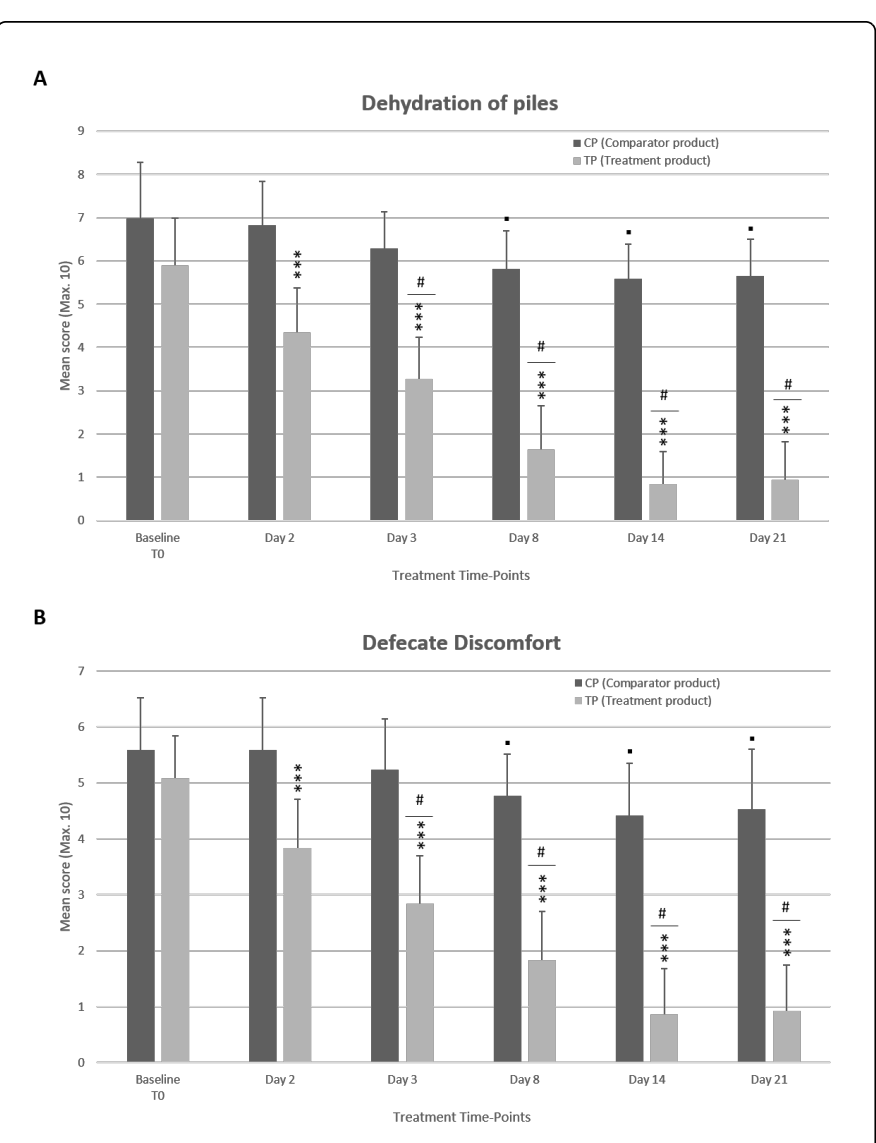

Figure 7: Effect of Pileseptine-e (TP) on the dehydration of piles (A) and defecation discomfort (B) scores quantified on a 0 (no symptom) to 10 (severe symptom) rating scale at the start of the treatment (baseline), on days $2,3,8$, and 14 as well as at the end of the follow-up period (day 21) in the CP (black bars) and in the TP (gray bars) groups. Values presented as Mean \pm S.D.. Statistical significance (SS) is indicated by ${ }^{* *} \mathrm{p}<0.001$ for TP compared to CP at the same time point, and $-\mathrm{p}<0.05$ compared to baseline values for $\mathrm{CP}, \# \mathrm{p}<0.05$ compared to baseline values for TP.

\section{Side effects}

Almost all patients reported a slight burning / itching sensation during the first 1-2 minutes following product application, along with a strong exudation of liquid from the hemorrhoidal surface, particularly during the initial $1 \mathrm{~h}$ post application. The initial itching sensation is probably related to the strong osmotic effects of the TP, causing transient cellular dehydration which is immediately compensated by circulatory liquid. No other undesired effects were observed by the patients or the investigators, either in the $\mathrm{CP}$ or the TP group. Similarly, there were no systemic side-effects indicating that the product Pileseptine-e act exclusively topically on the surface of the lesion.

\section{Discussion}

Hemorrhoids and hemorrhoidal lesions mainly concern deep blood vessels compressed at their neck, leading to the formation of an edematous vascular lesion. As veins are more prone to compression, the return venous flow becomes diminished, and as there is no natural physiological emergency mechanism to release the venous flow, the chronically dilated venous plexus externally appears as swollen hemorrhoids [12].

Tissue damage induces an inflammatory response, with an abundance of T-lymphocytes, macrophages, neutrophils, monocytes, mast cells and dendritic cells releasing both anti- and proinflammatory cytokines onto the hemorrhoidal surface [9]. When vascular damage and tissue swelling are not rapidly suppressed, this leads to an inflammatory cascade, the concentration of proinflammatory cytokines largely exceeding that of anti-inflammatory cytokines, and the healing process being totally halted $[9,10]$.

To treat such pathology, two pre-requisites become naturally evident: first, remove the edema, and secondly, minimize the concentration of contaminants on the affected surface, so as to allow natural hemorrhoidal regression and healing. Reducing edema can only be done by accelerating the venous blood circulation while restricting blood entry into the swollen hemorrhoidal tissue, or by attracting the edematous liquid out from the swollen vessels, without further damaging the tissue and exacerbating the inflammatory reaction.

In the absence of any topical anti-edematous, anti-inflammatory, and simultaneously tissue repairing therapy, the main goal of currently available medical treatment is to control acute symptoms of hemorrhoids rather than to cure the underlying cause of hemorrhoids [13]. There are several modern drugs and traditional medicine used which are available in a variety of format including pill, suppository, cream and wipes [5,14]. However, the published literature lacks strong evidence supporting the true efficacy of topical treatment for symptomatic hemorrhoids. For an oral preparation, flavonoids are the most common phlebotonic agent used for treating hemorrhoids [1]. It is probable that flavonoids could increase vascular tone and may have anti-inflammatory effects [3] when administered orally over a longer period of time but these effects can't be strong on the hemorrhoidal surface.

Unfortunately, there is no drug to selectively enhance venous return flow only, and there is no cell-friendly, non-irritant and non-cytotoxic product or method to remove liquid from an edematous lesion [7]. It is also not possible to use a single chemical and patentable substance as there is no single molecule that can block all the cytokines present on the hemorrhoidal surface $[11,15]$. It is important to note that the presence of any non-cell-friendly chemical in the vicinity of cells totally stops the healing process. Currently only saline solution is used as a natural, non-cytotoxic treatment to keep the hemorrhoidal surface hydrated and to minimize the pain. Unfortunately, due to its low osmotic activity, saline solution is immediately diluted and loses osmotic properties $[12,13,16,17]$. This is the reason why saline solution was used in this study as "comparator product".

Pileseptine-e is a filmogen, osmotically active solution made from glycerol, called VB-Gy $[15,16]$. Application of such a filmogen solution over an edematous tissue attracts the underlying hypotonic liquid, thereby reducing the volume of the lesion and keeping the tissue or lesion hydrated, which in turn reduces the pain, irritation, and helps reduce the concentration of all the contaminants present on its surface without any local or systemic toxicity. Glycerol equally possesses relatively good antiseptic properties, is derived from natural sources, it is non-toxic and non-irritant to the cells and tissues, and is transparent. It is not discarded that, despite the lack of biological 
Citation: Shrivastava L, Borges GDS, Shrivastava R (2018) Clinical Efficacy of a Dual Action, Topical Anti-edematous and Anti-inflammatory Device for the Treatment of External Hemorrhoids. Clin Exp Pharmacol 8: 246. doi:10.4172/2161-1459.1000246

Page 7 of 7

interaction with the cells, the plant polymer containing Pilecyanidin-e premix incorporated in VB-Gy to render it filmogen, may equally bind with the free-floating protein molecules on the hemorrhoidal surface. This binding would have reduced the concentration of proinflammatory cytokines, which are proteins in their nature, on the hemorrhoidal surface, followed by their mechanical removal through osmotic liquid flow. The aim of this study was therefore to evaluate the anti-edematous and healing properties of Pileseptine-e as a natural \& topical treatment, in order to provide a favorable ground for natural recovery process to occur.

These results are extremely encouraging and are considered to be related to the strong osmotic properties of Pileseptine-e which forms a hypertonic film over the hemorrhoidal surface and creates a strong outward exudation of hypotonic liquid through the semi-permeable hemorrhoidal membrane, which in consequence reduces the size of the lesion.

No side effects were observed in any of the patients, except for the slight and transient feeling of warmth irritation during the 1st few minutes.

Pileseptine-e, being a topical anti-inflammatory in addition to antioedematous should open a totally new therapeutic approach of treating external hemorrhoids.

\section{Acknowledgment}

This clinical study was commissioned and funded by the NATURVEDA SAS Laboratory.

\section{Conflict of Interest}

The authors have declared that no conflict of interest exists. Gisela Borges is an employer of NATURVEDA SAS but only participated on the revision of the manuscript.

\section{References}

1. Lohsiriwat V (2015) Treatment of hemorrhoids: A coloproctologist's view. World J Gastroenterol 21: 9245-9252.
2. Lohsiriwat V (2013) Approach to Hemorrhoids. Curr Gastroenterol Rep. 15: 332 .

3. Misra MC, Imlitemsu (2005) Drug treatment of haemorrhoids. Drugs 65: 1481-1491.

4. Tafti LD, Shariatpanahi SM, Damghani MM, Javadi B (2017) Traditional Persian topical medications for gastrointestinal diseases Iran J Basic Med Sci 20: 222-241.

5. Hardy A, Cohen CRG (2014) The acute management of haemorrhoids. Ann R Coll Surg Engl. 96: 508-511.

6. Dinarello CA (2010) Anti-inflammatory Agents: Present and Future. Cell 140: 935-950.

7. Shemesh EI, Kodner IJ, Fry RD, Neufeld DM (1987) Severe complication of rubber band ligation of internal hemorrhoids. Dis Colon Rectum 30: 199-200.

8. MacRae HM, McLeod RS (1995) Comparison of hemorrhoidal treatment modalities. A meta-analysis. Dis Colon Rectum 38: 687-694.

9. Shrivastava R (2005) Inventor; Naturveda, assignee. Non-solid composition for local application. United States patent US 6,964,783.

10. Srivastava A, Yadav SK, Yachha SK, Thomas MA, Saraswat VA, et al. (2011) Pro-inflammatory cytokines are raised in extrahepatic portal venous obstruction, with minimal hepatic encephalopathy. J Gastroenterol Hepatol 26: 979-986.

11. Shrivastava R, Shrivastava L, Shrivastava R (2017) Inventors. Composition for topical application comprising glycerol and tannins. United States patent application US 15/426,287.

12. Hammel HT, Schlegel WM (2005) Osmosis and Solute-Solvent Drag: Fluid Transport and Fluid Exchange in Animals and Plants. Cell Biochem Biophys 42: 277-345.

13. Hall JF (2013). Modern Management of Hemorrhoidal Disease. Gastroenterol Clin North Am 42: 759-772.

14. Kiil F (1989) Molecular mechanisms of osmosis. Am J Physiol 256: 801-808.

15. Tabas I, Glass CK (2013) Anti-inflammatory therapy in chronic disease: challenges and opportunities. Science 339:166-172.

16. Spitzbarth I, Baumgärtner W, Beineke A (2012) The role of pro- and antiinflammatory cytokines in the pathogenesis of spontaneous canine CNS diseases. Vet Immunol Immunopathol 147: 6-24.

17. Maspi N, Abdoli A, Ghaffarifar F (2016) Pro- and anti-inflammatory cytokines in cutaneous leishmaniasis: a review. Pathog Glob Health 110: 247-260. 